Liu A, Li ZX, Liu GF et al. Privacy-preserving task assignment in spatial crowdsourcing. JOURNAL OF COMPUTER SCIENCE AND TECHNOLOGY 32(5): 905-918 Sept. 2017. DOI 10.1007/s11390-017-1772-5

\title{
Privacy-Preserving Task Assignment in Spatial Crowdsourcing
}

An Liu ${ }^{1,2}$, Member, $C C F$, Zhi-Xu Li ${ }^{1, *}$, Member, $C C F$, Guan-Feng Liu ${ }^{1}$, Member, $C C F$ Kai Zheng ${ }^{1,3}$, Member, CCF, Min Zhang ${ }^{1}$, Member, CCF, Qing Li ${ }^{4}$, Senior Member, IEEE and Xiangliang Zhang ${ }^{2}$, Member, IEEE

${ }^{1}$ School of Computer Science and Technology, Soochow University, Suzhou 215006, China

${ }^{2}$ King Abdullah University of Science and Technology, Thuwal 23955, Saudi Arabia

${ }^{3}$ Beijing Key Laboratory of Big Data Management and Analysis Methods, Beijing 100872, China

${ }^{4}$ Department of Computer Science, City University of Hong Kong, Hong Kong, China

E-mail: \{anliu, zhixuli, gfliu, zhengkai, minzhang\}@suda.edu.cn; itqli@cityu.edu.hk xiangliang.zhang@kaust.edu.sa

Received March 1, 2017; revised August 11, 2017.

\begin{abstract}
With the progress of mobile devices and wireless networks, spatial crowdsourcing (SC) is emerging as a promising approach for problem solving. In SC, spatial tasks are assigned to and performed by a set of human workers. To enable effective task assignment, however, both workers and task requesters are required to disclose their locations to untrusted SC systems. In this paper, we study the problem of assigning workers to tasks in a way that location privacy for both workers and task requesters is preserved. We first combine the Paillier cryptosystem with Yao's garbled circuits to construct a secure protocol that assigns the nearest worker to a task. Considering that this protocol cannot scale to a large number of workers, we then make use of Geohash, a hierarchical spatial index to design a more efficient protocol that can securely find approximate nearest workers. We theoretically show that these two protocols are secure against semi-honest adversaries. Through extensive experiments on two real-world datasets, we demonstrate the efficiency and effectiveness of our protocols.
\end{abstract}

Keywords spatial crowdsourcing, spatial task assignment, location privacy, mutual privacy protection

\section{Introduction}

The ubiquity of high-quality wireless networks such as $4 \mathrm{G}$ and the popularity of smart devices equipped with powerful chips and useful sensors such as GPS have led to a variety of new application areas. One of the most promising platforms among them is spatial crowdsourcing $(\mathrm{SC})^{[1]}$ where spatial tasks (tasks related to a location) are assigned to and performed by human workers. The workers must physically move to the designated locations to perform corresponding tasks. A wide variety of spatial tasks are emerging nowadays, ranging from transportation (1) to traffic monitoring(2), to food delivery ${ }^{3}$, to handyman service (4), just to name a few. A typical workflow of spatial crowdsourcing, for example, in the FAVOR ${ }^{3}$ platform, begins with a food delivery order posted by a task requester, followed by a task assignment procedure during which an available worker (also called runner in FAVOR ${ }^{3}$ ) is selected to pick up the food at the specified restaurant and deliver it to the requester's home.

Currently, there are two common ways for task as-

Regular Paper

Special Section on Crowdsourced Data Management

This work was partially supported by King Abdullah University of Science and Technology (KAUST) and the National Natural Science Foundation of China under Grant Nos. 61572336, 61632016, 61402313, 61572335, and 61472337.

* Corresponding Author

(1) https://www.uber.com, August 2017.

(2) https://www.waze.com, August 2017.

(3) https://favordelivery.com, August 2017.

(4) https://www.taskrabbit.com, August 2017.

(C)2017 Springer Science + Business Media, LLC \& Science Press, China 
signment in SC (spatial crowdsourcing) ${ }^{[1]}$ : server assigned tasks (SAT) and worker selected tasks (WST). In the SAT mode, workers are required to authorize a spatial crowdsourcing server (SC-server) to continuously collect their physical location information. Upon receiving a spatial task, the $\mathrm{SC}$-server assigns to it suitable workers based on some business rules, for example, some nearby workers. However, the SC-server may not be trusted, and thus it is common for workers to raise privacy concerns as the collected location data may be shared with, rent or sold to the third parties. Based on these location or trajectory data, an adversary can stage a broad spectrum of attacks against individuals, such as physical surveillance and stalking, identity theft, and breach of sensitive information, for instance, home address, health condition, and lifestyle habits. Hence, worker location protection is an extremely important aspect of SC with SAT mode. In the WST mode, workers can choose any task without the coordination with the SC-server, and their locations are not disclosed to it. Accordingly, WST is more privacy-friendly than SAT from the workers' perspective. However, this comes at the expense of striping the SC-server of its control over the allocation of spatial tasks as a whole, which is not necessarily the ultimate objective of the SC-server ${ }^{[1]}$.

Moreover, location is also a kind of private information for task requesters. As an example, people suffering from health problems at home can ask for help by SC; however, the direct publication of their health problems together with their home addresses clearly breaches their privacy. Hence, task location should also be protected in the course of SC, no matter which task assignment mode is used.

Task assignment in SC has been extensively studied as an optimization problem recently. Given some temporal, budget, or skill constraints, these studies try to maximize, for example, the number of assigned tasks on the server side ${ }^{[1]}$, the number of workers' self-selected tasks on the client side ${ }^{[2]}$, the reliability-and-diversity score of assignment ${ }^{[3]}$, and the budget-minus-cost score of assignment ${ }^{[4]}$. However, all these studies fail to protect the privacy of either workers or task requesters.

On the other hand, a lot of efforts ${ }^{[5-10]}$ have been made towards location privacy protection in the scenario of location-based service (LBS). Earlier studies aim at enabling users to obtain results of location-based queries without revealing their exact locations to LBS providers. Recent studies also take into account privacy requirements of LBS providers (e.g., they do not allow others to know their private location data) and try to forbid users to learn anything more than query results. In $\mathrm{SC}$, however, worker location is not the private data of the SC-server, but rather the sensitive information that workers want to hide from the SC-server. This difference calls for new privacy-preserving models for task assignment in SC.

Recently there have also been some advances in privacy-preserving task assignment in SC applications $^{[11-14]}$. In [11-12], workers' locations are collected and perturbed by a trusted party which injects calibrated noises into raw data according to differential privacy $(\mathrm{DP})^{[15]}$. Upon receiving a spatial task, the SCserver queries the disguised location data to determine a region that is very likely to contain sufficient workers nearby the task. Workers in this region are notified about this task and they can decide whether or not to perform it. The proposed solution in this pioneering work, however, has two weaknesses. Firstly, it only takes into account worker privacy and fails to protect task location. Secondly, it works on a strong assumption that there is a trusted party called cellular service provider that has access to all workers' locations. This is justified by the observation that the cellular service provider could use cell tower triangulation to identify workers' locations. In fact, this justification is not very compelling in practice, as the accuracy of this method is generally between $150 \sim 300$ meters $^{[16]}$. On the other hand, if cellular service provider has the authority to collect workers' locations identified by GPS module equipped in smart devices, the accuracy will be much better and is generally between $1 \sim 5$ meters $^{[16]}$. In [13], workers' locations are encrypted by Paillier ${ }^{[17]}$ and indexed by an SKD tree, a newly designed data structure which eliminates potential information leakage of normal KD-tree caused by its public splitting dimension. However, workers are not static and they often move from one place to another, thereby the SKD tree needs to be updated frequently. Unfortunately, the update operation is very time-consuming especially when there are a large number of workers, which makes it unsuitable for large-scale real-time SC applications. The protocol proposed in [14] also takes into account worker velocity during task assignment, thus the result is more effective in practice. However, it still suffers from computation time issue and cannot scale to large SC applications.

In this paper, we tackle the problem of efficiently assigning workers to tasks in SC such that the location privacy for both workers and task requesters is 
protected without the help of a trusted third party. We first design a secure protocol that assigns the nearest worker to a task through the combination of two cryptographic techniques, that is, the Paillier encryption scheme ${ }^{[17]}$ and Yao's protocol (also known as Yao' garbled circuits) ${ }^{[18-19]}$. It is worth noting that we do not utilize some well-known cryptographic tools such as fully homomorphic encryption (FHE) ${ }^{[20-21]}$ and SMIN $^{[22]}$ due to their prohibitive computation cost. Instead, we divide the task assignment problem into two sub-tasks: Euclidean distance computation and integer comparison. We solve the former task by Paillier and make the result additively secret shared so that the latter task can be performed by Yao's protocol. Considering that the exact protocol cannot scale to a large number of workers, we propose further a protocol that efficiently finds approximate nearest workers. The efficiency improvement benefits from a hierarchical spatial index Geohash, and the security is guaranteed by the integration of Hash-ElGamal encryption scheme into the search process based on Geohash. Hash-ElGamal is a mature cryptographic tool whose security has been formally proved, but it cannot support Euclidean distance computation and comparison directly. Using a special spatial index, however, this complex computation is approximated to simple computation on bit strings, which is another contribution made by this paper.

The rest of the paper is organized as follows. Section 2 presents problem statement and Section 3 summaries the cryptographic tools used in this paper. Our basic protocol that finds exact solution is presented in Section 4. We propose a more efficient protocol that finds approximate solutions in Section 5. Section 6 reports the experimental results. Finally, Section 7 reviews the related work and Section 8 concludes the paper.

\section{Problem Statement}

\subsection{System Model}

We consider the problem of privacy-preserving task assignment in the SAT mode. Specifically, a task requester (TR) creates a spatial task $s$ that needs to be performed at location $l_{s}=\left(x_{s}, y_{s}\right)$ where $x_{s}$ and $y_{s}$ are longitude and latitude of $l_{s}$, respectively. Each worker $w_{i}$ is associated with an ID $i$ and a location $l_{i}=\left(x_{i}, y_{i}\right)$ where he/she is currently located. An SC-server is in charge of assigning nearby workers to $s$ based on their locations. Generally the nearest worker should be selected to perform $s$. During this procedure, both work- ers and the TR want to keep their locations private. Fig.1 depicts the proposed privacy-preserving SC system, where the SC-server performs privacy-preserving task assignment based on disguised locations, with the cooperation of a crypto-service provider (CSP). Note that the SC-server and the CSP constitute a dualserver architecture that is a common secure multi-party computation setting. Though both parties in this setting are required to involve in the computation (i.e., task assignment in spatial crowdsourcing), users can be relieved from heavy computation burden. Therefore, this setting is not only used in secure spatial crowdsourcing ${ }^{[13]}$ but also widely accepted in a variety of application domains, such as, secure $K \mathrm{NN}$ search ${ }^{[22]}$, secure trajectory computation ${ }^{[23]}$, and secure skyline query processing ${ }^{[24]}$. Unlike the model in [11] that assumes the existence of a trusted third party, our model only requires the CSP be semi-honest (also known as honest-but-curious, and will be briefly introduced in Subsection 2.2), which is more reasonable in practice.

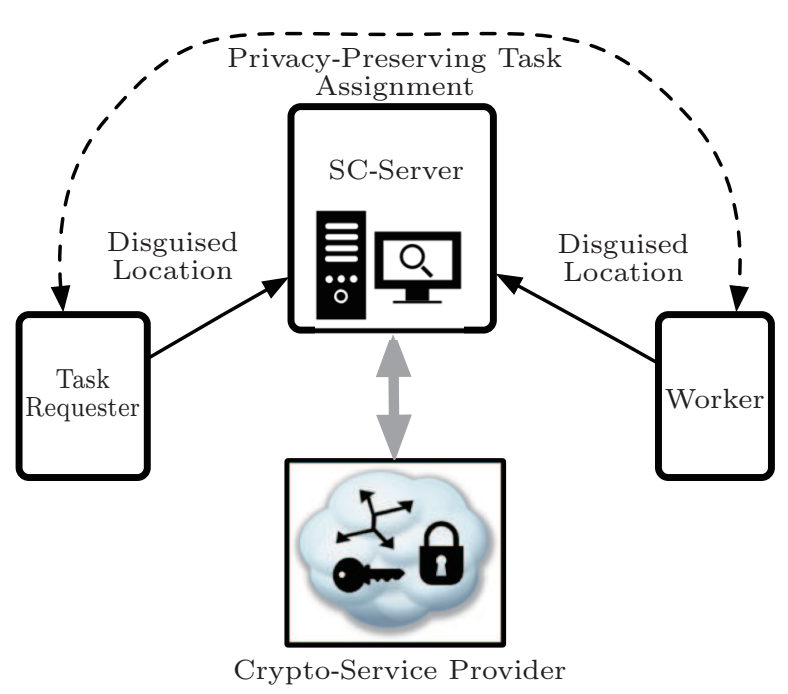

Fig.1. Privacy-preserving SC system.

\subsection{Threat Model and Security Definition}

In this paper, we adopt semi-honest adversaries without collusion, a common threat model used in different domains ${ }^{[13,22-24]}$. Specifically, we first assume all parties are semi-honest ${ }^{[25]}$, that is, they follow a protocol exactly as specified, but may try to learn as much as possible about other parties' private input from what they see during the protocol's execution. Note that although secure protocols against malicious adversaries exist, they are far too inefficient to implement and be used in practice. On the contrary, secure proto- 
cols against semi-honest adversaries are not only useful in practice but also the foundation of designing secure protocols against malicious adversaries. We further assume that the SC-server and the CSP do not collude. As emphasized in [26], the assumption of noncollusion between two well-established companies (i.e., the two parities in the dual-server setting) is reasonable as the collusion will damage their reputation and consequently reduce their revenues. A formal definition of security against semi-honest adversaries can be described as follows ${ }^{[25]}$.

Definition 1 (Security Against Semi-Honest Adversaries). Suppose that protocol $\mathscr{P}$ asks $\mathcal{A}$ to compute (and output) the function $f_{\mathcal{A}}(x, y)$, and asks $\mathcal{B}$ to compute (and output) $f_{\mathcal{B}}(x, y)$, where $x, y$ are the inputs of $\mathcal{A}$ and $\mathcal{B}$, respectively. Let view $w_{\mathcal{A}}(x, y)$ (resp. view $w_{\mathcal{B}}(x, y)$ ) represent $\mathcal{A}$ 's (resp. $\mathcal{B}$ 's) view during an execution of $\mathscr{P}$ on $(x, y)$. In other words, if $\left(x, r_{\mathcal{A}}\right)$ (resp. $\left.\left(y, r_{\mathcal{B}}\right)\right)$ denotes $\mathcal{A}$ 's (resp. $\mathcal{B}$ 's) input and randomness, then:

$$
\begin{aligned}
& \operatorname{view}_{\mathcal{A}}(x, y)=\left(x, r_{\mathcal{A}}, m_{1}, \ldots, m_{t}\right), \\
& \operatorname{view}_{\mathcal{B}}(x, y)=\left(y, r_{\mathcal{B}}, m_{1}, \ldots, m_{t}\right),
\end{aligned}
$$

where $m_{i}(i \in\{1, \cdots, t\})$ denote the messages passed between the parties. Also let $O_{\mathcal{A}}(x, y)\left(\right.$ resp. $\left.O_{\mathcal{B}}(x, y)\right)$ denote $\mathcal{A}$ 's (resp. $\mathcal{B}$ 's) output. Then we say that protocol $\mathscr{P}$ is secure against semi-honest adversaries if there exist probabilistic polynomial time $(P P T)$ simulators $\mathcal{S}_{1}$ and $\mathcal{S}_{2}$ such that:

$$
\begin{aligned}
\left(\mathcal{S}_{1}\left(x, f_{\mathcal{A}}(x, y)\right), f_{\mathcal{B}}(x, y)\right) & \equiv\left(\operatorname{view}_{\mathcal{A}}(x, y), O_{\mathcal{B}}(x, y)\right) \\
\left(f_{\mathcal{A}}(x, y), \mathcal{S}_{2}\left(y, f_{\mathcal{B}}(x, y)\right)\right) & \equiv\left(O_{\mathcal{A}}(x, y), \operatorname{view}_{\mathcal{B}}(x, y)\right)
\end{aligned}
$$

where $\equiv$ denotes computational indistinguishability.

Based on the above discussion, we give the definition of privacy-preserving task assignment problem as follows.

Definition 2 (Privacy-Preserving Task Assignment Problem). Let $W=\left\{w_{1}, w_{2}, \cdots, w_{n}\right\}$ be a set of workers. Given a spatial task s, the privacy-preserving task assignment problem is to assign the nearest worker $w_{i^{*}} \in W$ to $s$ such that neither the $S C$-server nor the CSP learns anything other than the task assignment result, that is, the ID of the nearest worker, $i^{*}$.

\section{Technical Preliminaries}

\subsection{Yao's Protocol}

Yao's protocol ${ }^{[18]}$ (a.k.a. garbled circuits) allows two semi-honest parties holding inputs $x$ and $y$, respec- tively, to evaluate an arbitrary function $f(x, y)$ without leaking any information about the inputs beyond what can be deduced by the function output. Viewing $f$ as a Boolean circuit, the evaluation of $f$ is realized by computing the circuit gate by gate, from the input wires to the output wires. Specifically, one party called sender constructs a garbled circuit and sends it to the other party called receiver. Every wire in the circuit is associated with two random cryptographic keys such that one represents 0 and the other represents 1 . Each gate in the circuit is associated with a garbled truth table which maps the value of two input wires (i.e., two random keys) to the value of the output wire (also a random key). Given two input-wire keys of a gate, it is only possible to compute the output value that corresponds to the output of the gate based on the garbled truth table. The receiver obtains the input-wire keys using oblivious transfer, computes the circuit as described, and produces the output. Due to space limitation, readers can refer to [19] for more details about Yao's protocol, including a complete security proof.

\subsection{Paillier Cryptosystem}

Paillier $^{[17]}$ is a public-key cryptosystem whose security is based on an assumption related (but not known to be equivalent) to the hardness of factoring. It consists of the following three algorithms.

- Key Generation. Choose two distinct large random primes $p, q$ and compute $N=p q$. Choose an element $g \in \mathbb{Z}_{N^{2}}^{*}$. The public key $k_{p}$ is $(N, g)$ and the secret key $k_{s}$ is $(p, q)$.

- Encryption $E$. Let $m$ be a message in $\mathbb{Z}_{N}$. It is encrypted by selecting a random number $r$ in $\mathbb{Z}_{N}^{*}$ and computing

$$
c=E(m)=g^{m} r^{N} \bmod N^{2} .
$$

- Decryption D. Ciphertext $c$ is decrypted by computing

$$
m=D(c)=\frac{\left(\left(c^{\lambda} \bmod N^{2}\right)-1\right) / N}{\left(\left(g^{\lambda} \bmod N^{2}\right)-1\right) / N} \bmod N,
$$

where $\lambda=\operatorname{lcm}(p-1, q-1)$.

One of the most important properties of Paillier is homomorphic addition. Specifically, multiplying an encryption of $m_{1}$ and an encryption of $m_{2}$ results in an encryption of $m_{1}+m_{2}$, and raising an encryption of $m$ to a constant $k$ results in an encryption of $k m$, that is,

$$
\begin{aligned}
& E\left(m_{1}\right) E\left(m_{2}\right)=E\left(m_{1}+m_{2}\right), \\
& E(m)^{k}=E(k m) .
\end{aligned}
$$


Besides, Paillier is semantic secure, that is, an adversary cannot learn any partial information about the plaintext from the ciphertext. As a result, it is also a probabilistic encryption scheme, which means when encrypting the same message several times, it will produce different ciphertexts.

\subsection{Hash-ElGamal Cryptosystem}

Hash-ElGamal ${ }^{[27]}$ is a public-key cryptosystem whose semantic security is based on the decision DiffieHellman assumption. It consists of the following three algorithms.

- Key Generation. Let $\mathbb{G}=\langle g\rangle$ be a cyclic group of order $q$. Choose an integer $x$ in $\mathbb{Z}_{q}$ and compute $y=g^{x}$ $\bmod q$. The public key $k_{p}$ is $(g, y)$ and the secret key $k_{s}$ is $x$.

- Encryption E. A message $m \in\{0,1\}^{l}$ is encrypted by selecting some random $r \in \mathbb{Z}_{q}$ and computing $c=E(m)=\left(c_{1}, c_{2}\right)=\left(g^{r}, H\left(y^{r}\right) \oplus m\right)$ where $H: \mathbb{G} \rightarrow\{0,1\}^{l}$ is a cryptographic hash function.

- Decryption $D$. A ciphertext $c$ is decrypted by computing $m=D(c)=H\left(c_{1}^{x}\right) \oplus c_{2}$.

It is worth remarking that one can publicly mask a ciphertext $c$ with any chosen random mask $\mu$ as $c^{\prime}=\left(c_{1}^{\prime}, c_{2}^{\prime}\right)=\left(c_{1}, c_{2} \oplus \mu\right)$. Decrypting $c^{\prime}$ produces the masked message $m^{\prime}=m \oplus \mu$ as follows: $m^{\prime}=$ $H\left(c_{1}^{\prime x}\right) \oplus c_{2}^{\prime}=H\left(c_{1}^{x}\right) \oplus c_{2} \oplus \mu=m \oplus \mu$.

\section{Finding Exact Nearest Worker}

In this section, we present ETA, a secure exact task assignment protocol, which assigns the exact nearest worker $w_{i^{*}}$ to $s$ without disclosing anything but the ID $i^{*}$ to the SC-server and the CSP. The basic idea of ETA is to securely compute the Euclidean distance between $s$ and every worker by the Paillier cryptosystem, and to securely compare these distances by Yao's protocol. Our design rationale is as follows. First note that in theory, we can design an approach to securely compute and compare Euclidean distances just based on a fully homomorphic encryption (FHE) scheme ${ }^{[20-21]}$, but this will incur prohibitive computation cost which makes the approach being of limited practical significance. We therefore resort to partially homomorphic encryption schemes. Among them, Paillier has been shown for its efficiency to securely compute Euclidean distance in different application domains ${ }^{[22-23,28]}$. Further, we note that Yao's protocol is quite efficient for comparing $n$ l-bit integers as the size of the corresponding circuit is bounded by $O(n l)^{[29]}$. Therefore, we com- bine the Paillier cryptosystem with Yao's protocol to design a secure and efficient protocol to find the exact nearest worker. A detailed description of ETA is presented in Algorithm 1.

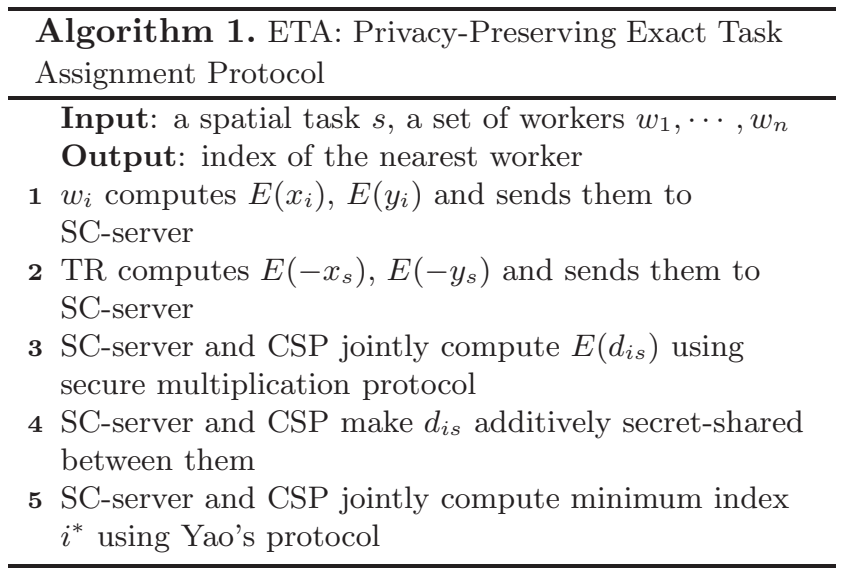

\subsection{Detailed Construction}

First, the CSP generates a pair of keys $k_{p}$ and $k_{s}$ for the Paillier cryptosystem. Every worker $w_{i}$ encrypts his/her location $l_{i}$ using $k_{p}$ and sends $E\left(x_{i}\right)$ and $E\left(y_{i}\right)$ to the SC-server. When the TR creates a task $s$, he/she also uses $k_{p}$ to encrypt $l_{s}$ and sends $E\left(-x_{s}\right)$ and $E\left(-y_{s}\right)$ to the SC-server. Upon receiving these encrypted values, the SC-server computes the squared Euclidean distance $d_{i s}$ between $l_{i}$ and $l_{s}$ by making use of Paillier's homomorphic addition property. Based on (1), $E\left(d_{i s}\right)$ equals $E\left(\left(x_{i}-x_{s}\right)^{2}\right) E\left(\left(y_{i}-y_{s}\right)^{2}\right)$ where $E\left(x_{i}-x_{s}\right)$ can be computed by multiplying $E\left(x_{i}\right)$ by $E\left(-x_{s}\right)$. To compute $E\left(\left(x_{i}-x_{s}\right)^{2}\right)$, the SC-server and the CSP jointly run a secure multiplication protocol ${ }^{[22]}$, whose basic idea is the observation on any given $m, r \in \mathbb{Z}_{N}$ : $m^{2}=(m+r)^{2}-2 m r-r^{2}$. Specifically, the SC-server generates a uniformly distributed random value $r$ from some appropriate range, computes $E(m+r)$ and sends it to the CSP. After obtaining $m+r$ through its secret key $k_{s}$, the CSP encrypts $(m+r)^{2}$ and sends it back to the SC-server. Finally, the SC-server holds $E\left(m^{2}\right)$ by computing $E\left((m+r)^{2}\right) E(m)^{-2 r} E\left(-r^{2}\right)$. By running the secure multiplication protocol $2 n$ times, the SCserver obtains $n$ distances between $s$ and $n$ workers, all in encrypted form.

To find the minimum index among $n$ values encrypted by Paillier, the SC-server first makes them additively secret shared between itself and the CSP. For every $E\left(d_{i s}\right)$, it chooses a random value $r$ from some appropriate range, adds the value to $E\left(d_{i s}\right)$ and sends $E\left(d_{i s}+r\right)$ to the CSP. After secret-sharing, the SC- 
server holds $n$ random values $r_{1}, \cdots, r_{n}$, and the CSP holds $n$ corresponding values $d_{1 s}+r_{1}, \cdots, d_{n s}+r_{n}$. Then, they compute the minimum index of $d_{1 s}, \cdots, d_{n s}$ through Yao's protocol. Fig.2 depicts the circuit that computes the minimum index of $n$ additively secretshared values. The difference between $d_{i s}+r_{i}$ and $r_{i}$ is computed by a subtraction circuit ${ }^{[29]}$ and then fed into a minimum circuit ${ }^{[29]}$ whose output is just the minimum index $i^{*}$. Note that the original minimum circuit in [29] also outputs the minimum, but we can hide it from both parties by simply removing corresponding output wires.

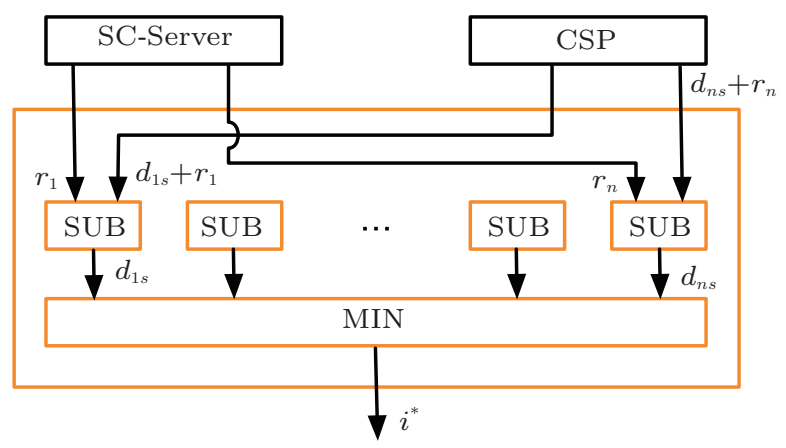

Fig.2. Circuit that computes minimum index of $n$ additively secret-shared values.

\subsection{Security and Complexity Analysis}

We first show the security of ETA.

Theorem 1. If the $S C$-server and the CSP do not collude, ETA is secure against semi-honest adversaries.

Proof. First note that ETA can be divided into three sequential phases: distance computation, secret sharing, and garbled circuit computation. In the first phase, we construct a simulator $S_{1}$ for the SC-server as follows: every time when the SC-server receives an encrypted message, $S_{1}$ randomly selects a number from some appropriate range and encrypts it. Clearly, the SC-server's view and $s_{1}$ 's view are computationally indistinguishable; otherwise this can be used to break the security of the Paillier cryptosystem. For CSP, we construct a simulator $S_{2}$ and let it pick a random number from some appropriate range when it receives an encrypted message. Though CSP has the secret key, any message it received has been masked by randomness of the SC-server's choice, which makes the CSP's view and $s_{2}$ 's view also computationally indistinguishable. Therefore, ETA is secure in the first phase. The security of secret sharing phase can be proved similarly.
The last phase of ETA follows exactly Yao's protocol, whose security proof is given in [19]. By noting these three phases are composed sequentially, it follows from the composition theory ${ }^{[25]}$ that ETA is secure against semi-honest adversaries.

Next, we analyze the complexity of ETA. As the computation and communication complexity of garbled circuits is much lower than that of homomorphic encryption ${ }^{[29]}$, we consider the cost of homomorphic encryption only. Table 1 summarizes the cost of ETA, where $E$ represents Paillier encryption, $D$ represents Paillier decryption, and $\kappa$ is the security parameter of Paillier. We assume all workers can perform computation (e.g., encryption and decryption) in parallel and can interact with the SC-server in parallel, thereby the cost on the user side can be reduced by computing for one user only. Besides, we ignore cheaper operations such as big integer multiplication and modular exponentiation. From Table 1, ETA is friendly to users and task requesters as they only need to perform two Paillier encryptions. For SC-server and CSP, the required number of Paillier encryptions is linear with the number of workers, and the constant is small.

Table 1. Complexity of ETA

\begin{tabular}{llll}
\hline & TR \& $w_{i}$ & SC-Server & CSP \\
\hline Computation & $2 E$ & $3 n E$ & $2 n E+n D$ \\
Communication & $2 \kappa$ & $(5 n+4) \kappa$ & $5 n \kappa$ \\
\hline
\end{tabular}

\section{$5 \quad$ Finding Approximate Nearest Workers}

While the SC-server's computation complexity in ETA is linear with the number of workers, ETA cannot be applied to real-time and large-scale SC scenarios because Paillier encryption is an expensive asymmetric operation. To enable real-world privacy-preserving SC applications, we propose in this section ATA, a more efficient privacy-preserving approximate task assignment protocol, by trading accuracy for efficiency. The objective of ATA is to efficiently compute a set of approximate nearest workers and assign them to $s$ in a privacypreserving manner. On one hand, Geohash (5), a hierarchical spatial index, is adopted to speed up approximate nearest workers search. On the other hand, Geohash codes are encrypted appropriately so that the nearest workers search can be carried out efficiently without disclosing private location information. A detailed description of ATA is presented in Algorithm 2.

(5)http://geohash.org/, August 2017. 


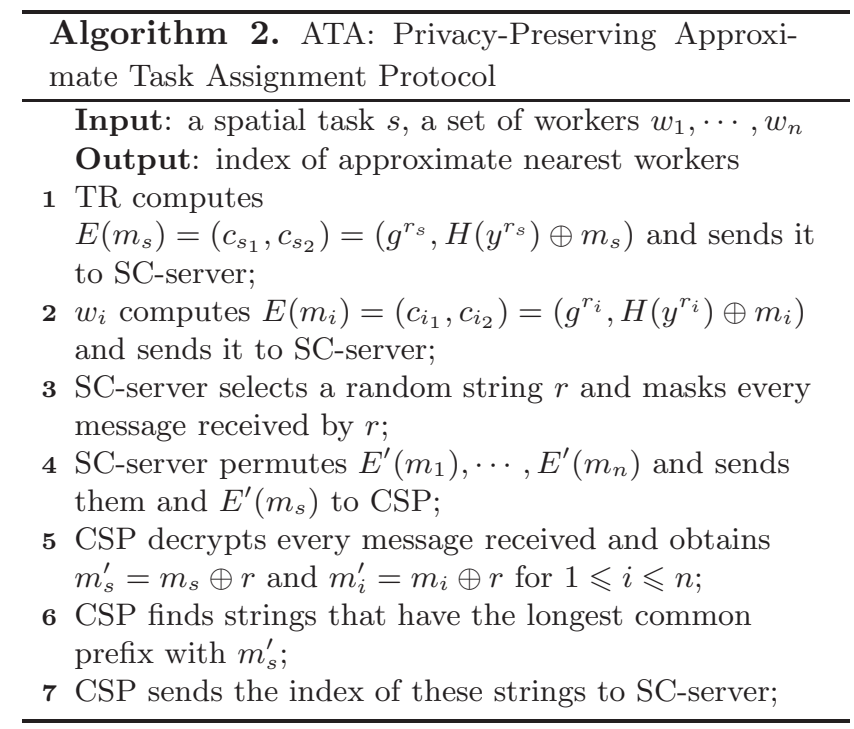

\subsection{Detailed Construction}

Geohash is a hierarchical, grid-based index of the earth where locations are represented by strings. A Geohash string (or code) is derived by interleaving bits obtained from latitude-longitude pairs and encoding them using base 32 character map. As depicted in Fig.3, the Geohash code of a Starbucks Coffee shop with coordinates $(31.315785,120.670518)$ is "wttf1uve". A Geohash code actually represents a rectangle (or bounding box) on the Earth. The longer the Geohash code is, the smaller the corresponding rectangle is. For example, the rectangle represented by "wttfluv" contains the rectangle represented by "wttf1uve". Also note that "wttf1uv" is just a prefix of "wttfluve", that is, spatial hierarchy can be defined by adding/removing characters at/from the end of Geohash codes. An interesting property of Geohash is that locations closer to each other are likely to have longer common prefixes in their Geohash codes. For example, in bit string representation of Geohash codes, A and B have 39-bit common prefix while $\mathrm{A}$ and $\mathrm{C}$ have 37 -bit common prefix. It is clear from Fig. 3 that $\mathrm{B}$ is nearer to A than $\mathrm{C}$, which coincides to the result obtained from common prefix.

In ATA, workers and the TR are required to give the SC-server their Geohash codes instead of coordinates. Computing the nearest workers then is equal to finding workers whose Geohash codes have the longest common prefixes with the Geohash code of $s$. While this computation is easy, more attention should be paid to the length of Geohash codes used in the course of task assignment. Generally, a short Geohash code represents a large area, which can be regarded as a cloaking area ${ }^{[30]}$ that provides certain privacy protection. Fig. 4 shows an example where the Geohash codes of $w_{1}, w_{2}$, and $s$ are "wttf0", "wttf1", and "wttf1", respectively. The area of the rectangle represented by "wttf0" is about 23.9 $\mathrm{km}^{2}$. From privacy perspective, knowing $w_{1}$ locates in such an area is much better than knowing his/her exact location. In this case, however, $w_{2}$ is considered to be the nearest worker based on common prefix, which is clearly a bad result. Therefore, long Geohash codes are preferred from effectiveness perspective.

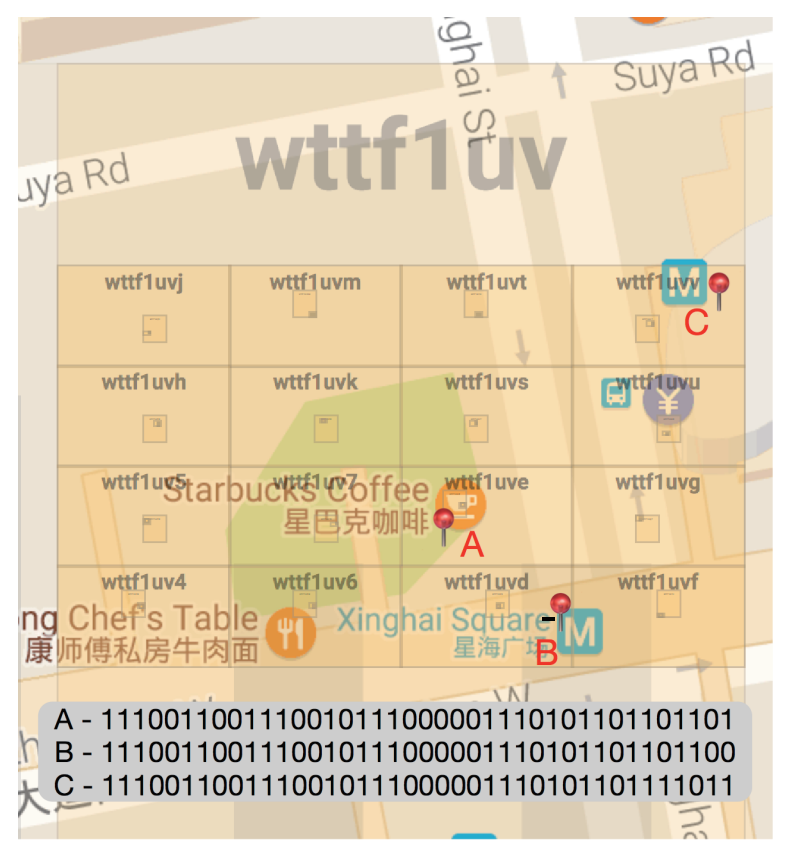

Fig.3. Geohash code example.

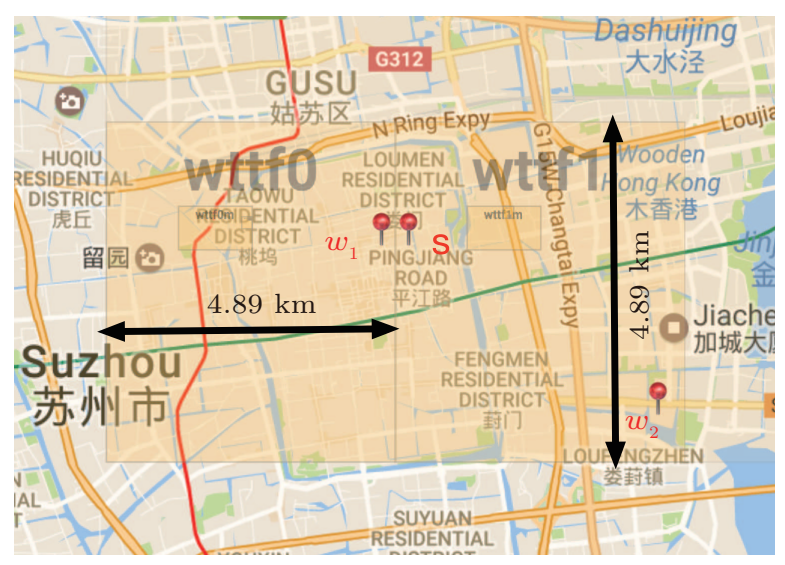

Fig.4. Proximity search based on Geohash codes.

When using long Geohash codes, say 10 Base 32 characters, the area of corresponding rectangles is less than $0.8 \mathrm{~m}^{2}$. Knowing workers located in such small 
rectangles clearly breaches their location privacy. Next, we give an efficient design to protect Geohash codes of workers and the TR.

From now on we shall consider Geohash codes as bit strings. Suppose $m_{1}, \cdots, m_{n}, m_{s}$ are the Geohash codes of $w_{1}, \cdots, w_{n}$ and $s$, respectively, and all of them have the same length $L$. The length of the longest common prefix between $m_{i}$ and $m_{s}$ is denoted by $l c p\left(m_{i}, m_{s}\right)$. For any $L$-bit string $r$, the following equation always holds:

$$
l c p\left(m_{i}, m_{s}\right)=l c p\left(m_{i} \oplus r, m_{s} \oplus r\right),
$$

due to the fact that $b_{1}=b_{2} \Leftrightarrow b_{1} \oplus b_{3}=b_{2} \oplus b_{3}$ and $b_{1} \neq b_{2} \Leftrightarrow b_{1} \oplus b_{3} \neq b_{2} \oplus b_{3}$ for any bits $b_{1}, b_{2}$ and $b_{3}$. From (2), the longest common prefix computation on the XORed Geohash codes has the same result as that on the original Geohash codes. Based on this property, we use Hash-ElGamal to encrypt original Geohash codes.

As shown in Algorithm 2, the TR obtains the Geohash code $m_{s}$ of task $s$, encrypts it using CSP's public key of Hash-ElGamal cryptosystem, and sends the ciphertext $E\left(m_{s}\right)=\left(c_{s_{1}}, c_{s_{2}}\right)=\left(g^{r_{s}}, H\left(y^{r_{s}}\right) \oplus m_{s}\right)$ to the SC-server. The Geohash codes of all workers are protected likewise. Upon receiving all encrypted Geohash codes, the SC-server selects a random $L$-bit string $r$, and masks every message received using $r$ as follows: $E^{\prime}\left(m_{s}\right)=\left(c_{s_{1}}, c_{s_{2}} \oplus r\right)$. For the sake of security, the SC-server also permutes the masked values before they are sent out. Using its secret key $x$, CSP can decrypt $E^{\prime}\left(m_{s}\right)$ to $m_{s}^{\prime}=m_{s} \oplus r$ as follows: $c_{s_{2}} \oplus r \oplus H\left(c_{s_{1}}^{x}\right)=H\left(y^{r_{s}}\right) \oplus m_{s} \oplus r \oplus H\left(\left(g^{r_{s}}\right)^{x}\right)=m_{s} \oplus r$. $E^{\prime}\left(m_{i}\right)$ can be decrypted likewise. After that, CSP finds strings in $m_{1} \oplus r, \cdots, m_{n} \oplus r$ that have the longest common prefix with $m_{s} \oplus r$. The index of these strings is then sent to the SC-server to complete the execution of ATA.

\subsection{Security and Complexity Analysis}

The security described in Definition 1 is so strong that sometimes it cannot be achieved by efficient protocols. In practice, an extra knowledge $K$ disclosure during an execution of protocol $P$ can be allowed (for efficiency) as long as it does not breach privacy in the sense that, even based on $K$, the probability that an adversary learns the private input of any party during an execution of protocol $P$ is negligible. With this modified (weaker) security definition, we analyze the security of ATA.
Theorem 2. As long as the $S C$-server and the CSP do not collude, ATA is weakly secure against semihonest adversaries.

Proof. Similar to the proof of Theorem 1, the computational indistinguishability for the SC-server originates from the semantic security of Hash-ElGamal that is based on the decision Diffie-Hellman assumption. The case of CSP is complex as it holds $n+1$ values $m_{1} \oplus r, \cdots, m_{n} \oplus r, m_{s} \oplus r$, which constitute the extra knowledge $K$. We will show this disclosure has negligible contribution to learning the private inputs $m_{1}, \cdots, m_{n}, m_{s}$. First note that random $r$ can be eliminated by XORing any two values, thus CSP can obtain $n$ values $m_{1} \oplus m_{s}, \cdots, m_{n} \oplus m_{s}$. Let $v_{i}=m_{i} \oplus m_{s}, v_{i j}$ be the $j$-th bit of $v_{i}$ and note that all strings have $L$ bits. CSP can construct an equation system: $\left\{m_{11} \oplus m_{s 1}=v_{11}, \cdots, m_{1 L} \oplus m_{s L}=v_{1 L}, \cdots\right.$, $\left.m_{n L} \oplus m_{s L}=v_{n L}\right\}$ which consists of $n L$ equations with $(n+1) L$ unknowns whose values are either 0 or 1 . It is clear that the system has $2^{L}$ solutions (by enumerating all possible values of $m_{s}$ ). Further note that these values are permuted by the SC-server, thus any permutation of each solution yields a different solution, that is, the equation system has totally $2^{L} n$ ! solutions, which makes the probability that CSP learns $m_{1}, \cdots, m_{n}, m_{s}$ based on $K$ negligible.

Table 2 shows the complexity of ATA where $E$ represents Hash-ElGamal encryption, $D$ represents HashElGamal decryption, $\kappa$ is the security parameter of Hash-ElGamal, and $L^{\prime}$ is the length of the digest of the cryptographic hash function used in Hash-ElGamal. We also assume that all workers can perform computation in parallel and can interact with the SC-server in parallel. We ignore cheap operations such as XOR of two bit strings, and thus the computation cost of SCserver is negligible (denoted as negl). Also note that the ciphertext of Hash-ElGamal consists of two parts, and thus its length equals $\kappa+L^{\prime}$. It is clear from Table 1 and Table 2 that ATA is more efficient than ETA. We will show this more specifically by experiments in the next section.

Table 2. Complexity of ATA

\begin{tabular}{llll}
\hline & TR \& $w_{i}$ & SC-Server & CSP \\
\hline Computation & $1 E$ & negl & $n D$ \\
Communication & $\kappa+L^{\prime}$ & $2(n+1)\left(\kappa+L^{\prime}\right)$ & $(n+1)\left(\kappa+L^{\prime}\right)$ \\
\hline
\end{tabular}




\section{Performance Evaluation}

\subsection{Experimental Setting}

We evaluate the performance of our protocols from three aspects: running time, communication cost, and accuracy. As discussed earlier, there are only a couple of studies ${ }^{[11-13]}$ studying privacy-preserving task assignment in SC applications, thereby we decide to compare our protocols with To et al.'s approach ${ }^{[11-12]}$ (denoted as DPTA since it is based on differential privacy). Note that ATA cannot guarantee to find the nearest worker, nor can DPTA. Hence, we take worker travel distance (WTD) proposed in [11] for accuracy evaluation. In particular, WTD equals the average of the sum of the distances between the task and the selected workers. For ETA, only one worker is selected, thereby WTD is just the distance between the worker and the task. Note that the performance of DPTA depends on the privacy budget $\epsilon$ for differential privacy, thereby we consider its performance under two representative values: $\epsilon=0.1$ for strong privacy guarantee and $\epsilon=1$ for weak privacy guarantee. We randomly generate 1000 tasks and report the average results.

We notice that differential privacy is clearly much computationally cheaper than public-key cryptosystems. However, it cannot protect data during computation (e.g., a trusted third party is allowed to hold all workers' private data); thus it is meaningless to compare our protocols (based on public-key cryptosystems) with DPTA (based on differential privacy) in terms of running time. In fact, we implemented DPTA and found that it only needs about $10 \mathrm{~ms}$ when performing task assignment for 1000 workers, which is clearly much faster than methods based on public-key cryptosystems. Besides, our efficiency study does not include the method proposed in [13] as, according to [13], the time of building an SKD-tree for 1000 workers is more than 100 seconds, whereas our protocol ATA only needs less than three seconds for task assignment among 1000 workers as we will show next. Therefore, we only evaluate the implementation of our protocols next, testing whether their overheads could be accepted in practice. We execute our protocols 10 times and report the average results.

We use two real-world datasets, Gowalla ${ }^{(6)}$ and Yelp ${ }^{(7}$, for performance evaluation. Gowalla contains the check-in history of users in a location-based social network. We select an area in California with the latitude from 33.720183 to 34.149932 and the longitude from -118.399999 to -117.900516 . This area has the check-ins of 5830 users who are assumed to be the workers in a spatial-crowdsourcing system. We take the location where a user has most check-ins as his/her current location, and assume that a spatial task can be created in any location that has check-ins. For Yelp, we select an area in Phoenix with the latitude from 33.205308 to 33.924407 and the longitude from -112.400283 to -111.218100 . This area has about 67000 users and 11200 businesses. Business locations are regarded as tasks while the location of a user is randomly selected from businesses on which she/he has a review.

Unless otherwise mentioned, our experiments are conducted on a machine with Intel Core i5-3470 $3.2 \mathrm{GHz}$ CPU and 8 GB RAM. We implement our protocol using the Bouncy Castle crypto package ${ }^{8}$ and the FastGC framework ${ }^{[31]}$ for running garbled circuits. The security parameters of Paillier and Hash-ElGamal are both set to 1024 . The default length of Geohash code is set to 50 bits. The code is written in Java and executed in JDK 1.8 .

\subsection{Experimental Results}

\subsubsection{Effect of the Number of Workers}

Fig.5 shows the running time of the proposed two protocols. As expected, ATA is much faster than ETA as the proximity search in ATA is based on highly efficient XOR operations over ciphertexts. For example, it only needs less than three seconds to make task assignment for 1024 workers on an ordinary computer. The running time of ETA increases rapidly as the number of workers increases, because a lot of expensive Paillier operations are performed in ETA. This is the cost to achieve strong privacy guarantee, that is, neither the SC-server nor the CSP can learn anything other than the final result of task assignment. To the contrary, ATA allows limited knowledge disclosure (but does not breach the individual privacy of workers and task requesters as proved earlier) to pursue usability in practice, for example, it is about 50 times faster than ETA when processing 1024 workers.

\footnotetext{
(6)https://snap.stanford.edu/data/loc-gowalla.html, August 2017.

(7) https://www.yelp.com/dataset_challenge, August 2017.

(8)https://www.bouncycastle.org/java.html, August 2017.
} 


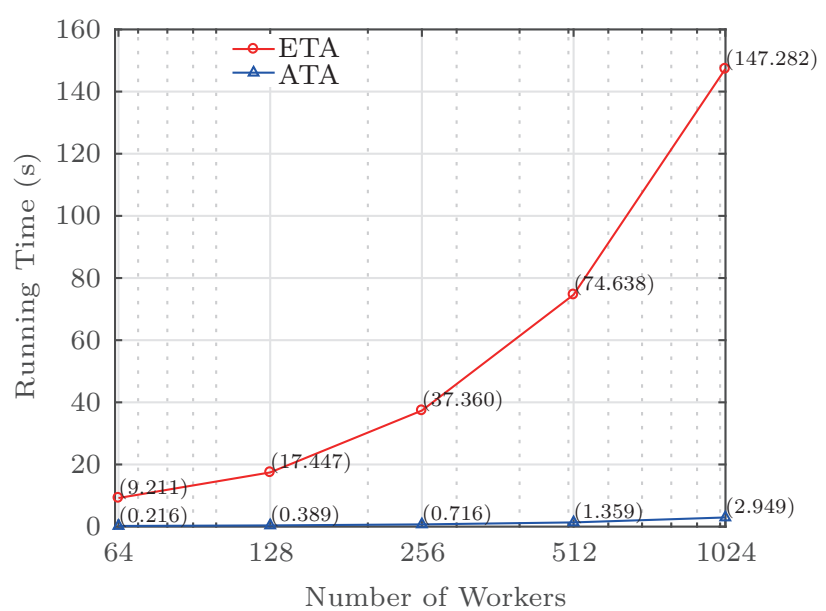

Fig.5. Effect of the number of workers on running time.

Fig.6 shows the communication cost of ETA and ATA. Overall, both protocols perform well for thousands of workers, as neither the SC-server nor the CSP in ETA needs to transfer more than 4.5 MB data when conducting a task assignment for 1024 workers. The communication costs for workers and task requesters are less than $1 \mathrm{~KB}$, thereby they are not shown in Fig.6. Moreover, ATA incurs less communication cost than ETA though the ciphertext of Hash-ElGamal is longer than that of Paillier for the same level of security. Thus, ATA is efficient not only in computation but also in communication, which makes it quite feasible in practice.

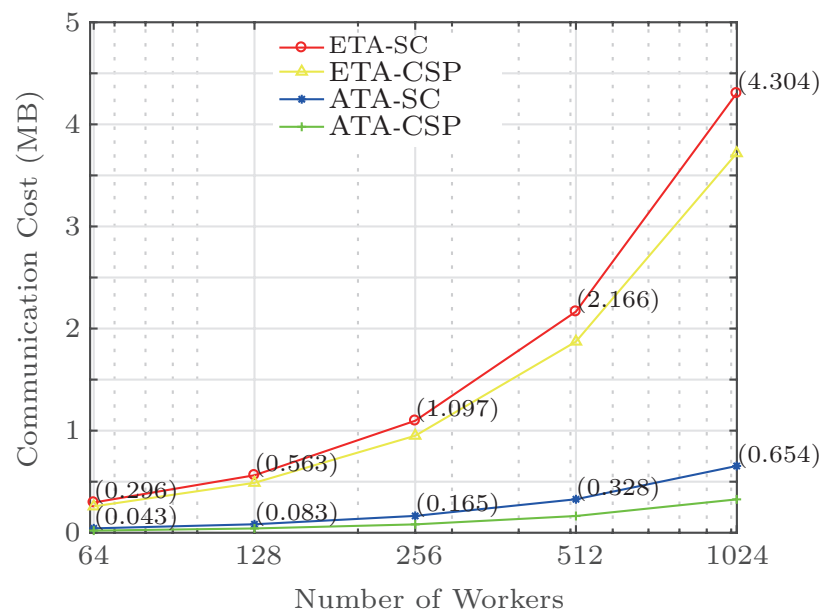

Fig.6. Effect of the number of workers on communication cost.

The complexity analysis of ATA in Table 2 shows the performance bottleneck of ATA lies in the decryption of $n$ ciphertexts performed by CSP. Fortunately, these decryptions are independent with each other and thus can be parallelized easily. To justify this claim, we run ATA on a server with four Intel Xeon E7-8860 $2.2 \mathrm{GHz}$ CPUs, each of which has 16 cores. In particular, the decryption operations of CSP are performed by 64 threads. Fig. 7 shows the performance of parallelized ATA. Even for a large task assignment problem with 8192 workers, the total running time is less than 1.3 seconds. We also measure the communication cost of ATA for a large number of workers. In Fig.8, we can see that the SC-server and the CSP only need to transfer about 5.2 $\mathrm{MB}$ and 2.6 MB data, respectively, for more than $8 \mathrm{k}$ workers. Clearly, the time for transferring this volume of data can be negligible in current networks. The above results demonstrate that ATA can scale to large real SC applications.

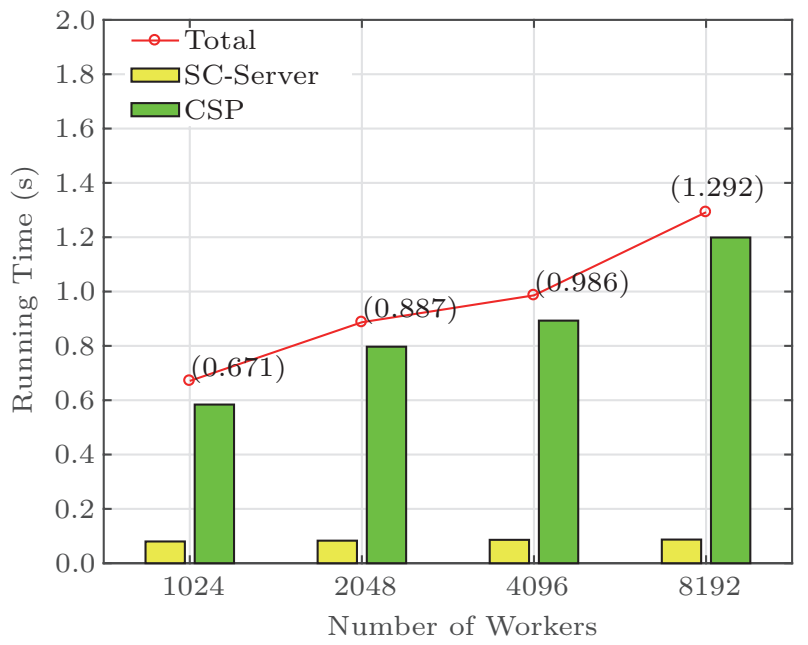

Fig.7. Running time of parallelized ATA.

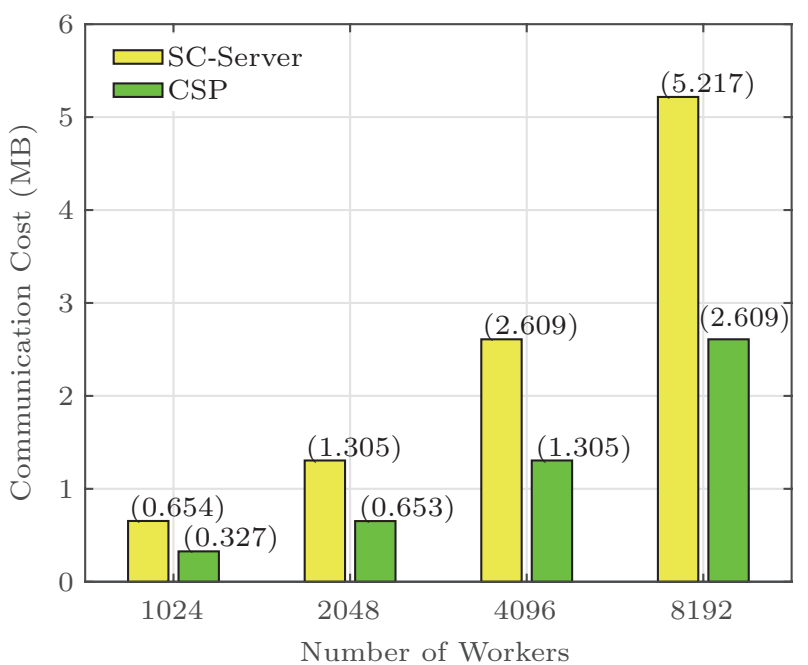

Fig.8. Communication cost of parallelized ATA. 


\subsubsection{Effect of Geohash Code Length}

From Table 2, the complexity of ATA does not depend on the length of Geohash codes. However, its length decides the size of the corresponding bounding box, which in turn decides the number of selected workers. Fig.9 shows worker travel distance of ATA on two real datasets. As expected, WTD decreases as the length of Geohash code increases. This is because long Geohash codes represent small bounding boxes, which contributes to the finding of workers that are indeed near to given tasks. It is interesting to note that ATA has lower WTD on Yelp than Gowalla since workers in Yelp are denser.

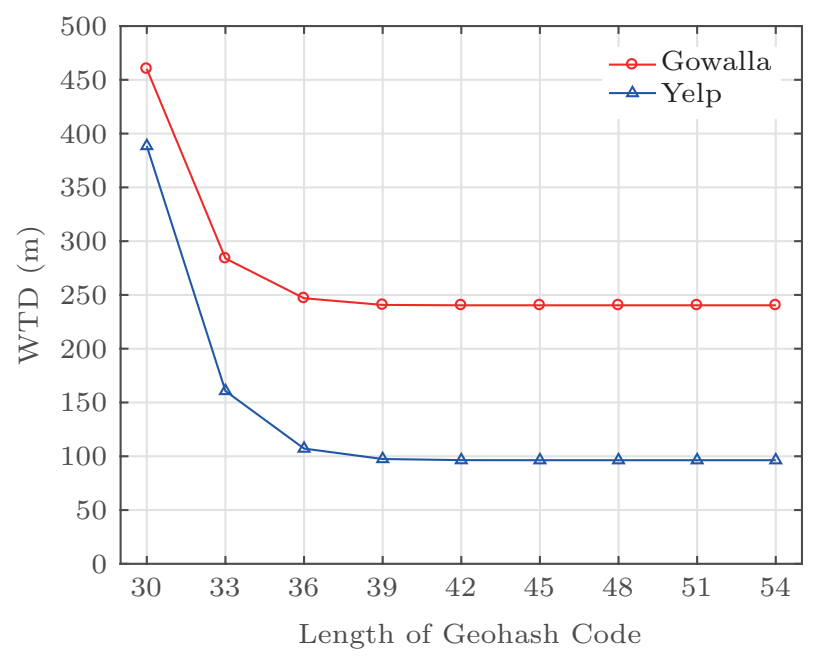

Fig.9. Effect of Geohash code length on WTD.

\subsubsection{Accuracy}

Table 3 shows the accuracy of different protocols on two real datasets. As ETA is an exact algorithm, it actually establishes the lower bound of WTD on different datasets. ATA cannot beat ETA due to its approximation nature, but outperforms DPTA on both datasets. Using ATA, the WTD is reduced by at most $75 \%$ compared with DPTA $(\epsilon=0.1)$ on Yelp, and by at least $42 \%$ compared with DPTA $(\epsilon=1)$ on Gowalla. Compared with the optimal result, workers only need to travel more than about 100 and 50 meters in Gowalla and Yelp, respectively, when ATA is used for privacypreserving task assignment.

Table 3. Average WTD by Different Methods (m)

\begin{tabular}{lrrcc}
\hline Dataset & ETA & \multicolumn{1}{c}{ ATA } & DPTA $(\epsilon=0.1)$ & DPTA $(\epsilon=1)$ \\
\hline Gowalla & 141.9 & $\mathbf{2 4 0 . 4}$ & 630.1 & 408.1 \\
Yelp & 54.9 & $\mathbf{9 6 . 3}$ & 382.9 & 262.2 \\
\hline
\end{tabular}

\section{Related Work}

As a special mode of crowdsourcing ${ }^{[32]}, \mathrm{SC}$ is a complex procedure that generally consists of four phases: task and workers registration, task assignment, answer aggregation, and response and quality control ${ }^{[33]}$. Here we only review the studies that are relevant to task assignment, referring readers to [33] for more results on other phases of SC. Kazemi and Shahabi ${ }^{[1]}$ proposed several heuristics to maximize the overall task assignment while conforming to the constraints of workers. Similarly, Deng et al. ${ }^{[2]}$ devised both exact and approximation algorithms to find a schedule for a worker such that the number of performed tasks by the worker is maximized. Spatial-temporal diversity and reliability are also taken into account in the course of task assignment. [3] shows task assignment with these constraints is NP-hard and proposes several approximation algorithms. In [4], efficient methods are designed to assign workers to complex tasks that require more than one skill. Tong et al. ${ }^{[34]}$ considered task assignment in online scenarios and proposed efficient algorithms with provable competitive ratio. Zheng and Chen ${ }^{[35]}$ tackled the problem of assigning tasks to workers such that mutual benefit is maximized. All these studies assume that workers are willing to give their private location information to the SC-server that is typically untrusted in practice. Our work complements these studies by tackling the privacy leakage problem in the phase of task assignment.

Location privacy protection has been studied extensively in recent years. Ghinita et al. ${ }^{[5]}$ adopted private information retrieval (PIR) to enable users to conduct approximate and exact nearest neighbor search without revealing their locations to the server. Paulet et al. ${ }^{[6-7]}$ combined PIR and oblivious transfer (OT) to achieve mutual privacy-preserving location-based queries. On one hand, the server is unable to know the location of users. On the other hand, users can only get a limited location data for their queries, thus protecting the server's private data. Liu et al. ${ }^{[10]}$ proposed a more efficient approach for this problem by utilizing two rounds of OT and showed the efficiency improvement can be realized at the expense of acceptable communication cost. Yi et al. ${ }^{[8]}$ presented a solution based on Paillier and Rabin cryptosystem for mutual privacy-preserving $k \mathrm{NN}$ query where $k$ is fixed. The solution is extended in [9] to support dynamic $k$ up to constant and sequential queries. However, these solutions cannot be applied to our scenario. This is because, in SC, worker location is not the private data of the SC-server, but rather the 
sensitive information that workers want to hide from the SC-server. There are also some studies focusing on privacy-preserving location-based queries over outsourced location data ${ }^{[36-37]}$, where the data owner and users sending queries are assumed to trust each other. In SC, however, there is no inherent trust relationship between task requesters and workers. To enable $k \mathrm{NN}$ query over encrypted data, Elmehdwi et al. ${ }^{[22]}$ proposed a set of protocols based on Paillier. While mutual privacy can be guaranteed due to the security of Paillier, the computation cost of these protocols are very expensive ${ }^{[23]}$. Thus, we cannot apply these protocols to directly solve large task assignment problems.

A number of studies have also reported privacy protection on a special SC, that is, participatory sensing, where workers (or participants) first collect data by using their mobile phones as sensors and then send these data to the SC-server for analysis. WST is the primary mode of task assignment in participatory sensing, thereby the most privacy disclosure comes from the step of data reporting. Techniques of protecting workers' privacy in this phase include anonymity which aims to break the links between workers' identities and their contributed data ${ }^{[38-39]}$, location cloaking by which workers replace their exact locations by cloaked spatial areas ${ }^{[40-41]}$, data perturbation which adds noise into workers' contributed data in a way that the statistical results over all workers' disguised data can be evaluated effectively ${ }^{[42]}$, and data aggregation which merges contributed data from different workers to obscure the mappings between workers' identities and the contributed data ${ }^{[43]}$. Unlike these studies, Kazemi and Shahabi ${ }^{[44]}$ focused on privacy-preserving task assignment in participatory sensing. Each worker first cloaks his/her location and range query among other $k-1$ workers. Then only a small group of workers is selected to submit their queries to the SC-server. These workers are also responsible for sharing query results to other workers according to their regions. The security of this method depends on the trust among workers and can thus be endangered when some workers would collude with the SC-server. On the contrary, our approach does not rely on the trust among workers or a trusted third party and is therefore more robust in practice.

\section{Conclusions}

In this paper, we studied privacy-preserving task assignment problem for spatial crowdsourcing. The challenge lies in mutual privacy-preserving, that is, workers are assigned to tasks in a way that location privacy for both workers and task requesters is preserved. We presented two protocols for this problem: ETA is an exact algorithm that is able to find the nearest worker, while ATA is a more efficient algorithm that finds approximate nearest workers. We theoretically analyzed security and complexity for both protocols. Extensive experiments on two real-world datasets showed the efficiency and effectiveness of our proposed protocols.

\section{References}

[1] Kazemi L, Shahabi C. GeoCrowd: Enabling query answering with spatial crowdsourcing. In Proc. the 20th Int. Conf. Advances in Geographic Information Systems, November 2012, pp.189-198.

[2] Deng D X, Shahabi C, Demiryurek U, Zhu L H. Task selection in spatial crowdsourcing from worker's perspective. GeoInformatica, 2016, 20(3): 529-568.

[3] Cheng P, Lian X, Chen Z, Fu R, Chen L, Han J S, Zhao J Z. Reliable diversity-based spatial crowdsourcing by moving workers. Proceedings of the VLDB Endowment, 2015, 8(10): 1022-1033.

[4] Cheng P, Lian X, Chen L, Han J S, Zhao J Z. Task assignment on multi-skill oriented spatial crowdsourcing. IEEE Trans. Knowledge and Data Engineering, 2016, 28(8): 2201-2215.

[5] Ghinita G, Kalnis P, Khoshgozaran A, Shahabi C, Tan K L. Private queries in location based services: Anonymizers are not necessary. In Proc. the ACM SIGMOD Int. Conf. Management of Data, June 2008, pp.121-132.

[6] Paulet R, Kaosar M G, Yi X, Bertino E. Privacy-preserving and content-protecting location based queries. In Proc. the 28th IEEE Int. Conf. Data Engineering, April 2012, pp.4453.

[7] Paulet R, Kaosar M G, Yi X, Bertino E. Privacy-preserving and content-protecting location based queries. IEEE Trans. Knowledge and Data Engineering, 2014, 26(5): 1200-1210.

[8] Yi X, Paulet R, Bertino E, Varadharajan V. Practical $k$ nearest neighbor queries with location privacy. In Proc. the 30th IEEE Int. Conf. Data Engineering, March 31-April 4 2014, pp.640-651.

[9] Yi X, Paulet R, Bertino E, Varadharajan V. Practical approximate $k$ nearest neighbor queries with location and query privacy. IEEE Trans. Knowledge and Data Engineering, 2016, 28(6): 1546-1559.

[10] Liu S S, Liu A, Zhao L, Liu G F, Li Z X, Zhao P P, Zheng K, Qin L. Efficient query processing with mutual privacy protection for location-based services. In Proc. the 21st Int. Conf. Database Systems for Advanced Applications, April 2016, pp.299-313.

[11] To H, Ghinita G, Shahabi C. A framework for protecting worker location privacy in spatial crowdsourcing. Proceedings of the VLDB Endowment, 2014, 7(10): 919-930. 
[12] To H, Ghinita G, Shahabi C. PrivGeoCrowd: A toolbox for studying private spatial crowdsourcing. In Proc. the 31st IEEE Int. Conf. Data Engineering, April 2015, pp.14041407.

[13] Liu B Z, Chen L, Zhu X Q, Zhang Y, Zhang C Q, Qiu W D. Protecting location privacy in spatial crowdsourcing using encrypted data. In Proc. the 20th Int. Conf. Extending Database Technology, March 2017, pp.478-481.

[14] Liu A, Wang W Q, Shang S, Li Q, Zhang X L. Efficient task assignment in spatial crowdsourcing with worker and task privacy protection. Geoinformatica, 2017. DOI: 101007/s10707-017-0305-2.

[15] Dwork C. Differential privacy: A survey of results. In Proc. the 5th Int. Conf. Theory and Applications of Models of Computation, April 2008.

[16] Hightower J, Borriello G. Location systems for ubiquitous computing. Computer, 2001, 34(8): 57-66.

[17] Paillier P. Public-key cryptosystems based on composite degree residuosity classes. In Proc. the 17th Int. Conf. Theory and Application of Cryptographic Techniques, May 1999, pp.223-238.

[18] Yao A C C. How to generate and exchange secrets. In Proc. the 27th Annual Symp. Foundations of Computer Science, October 1986, pp.162-167.

[19] Lindell Y, Pinkas B. A proof of security of Yao's protocol for two-party computation. Journal of Cryptology, 2009, 22(2): 161-188.

[20] Gentry C. Fully homomorphic encryption using ideal lattices. In Proc. the 41st Annual ACM Symp. Theory of Computing, May 31-June 2, 2009, pp.169-178.

[21] Gentry C, Halevi S. Implementing Gentry's fullyhomomorphic encryption scheme. In Proc. the 30th Annual Int. Conf. Theory and Applications of Cryptographic Techniques: Advances in Cryptology, May 2011, pp.129-148.

[22] Elmehdwi Y, Samanthula B K, Jiang W. Secure $k$-nearest neighbor query over encrypted data in outsourced environments. In Proc. the 30th IEEE Int. Conf. Data Engineering, March 31-April 4, 2014, pp.664-675.

[23] Liu A, Zheng K, Li L, Liu G F, Zhao L, Zhou X F. Efficient secure similarity computation on encrypted trajectory data. In Proc. the 31st IEEE Int. Conf. Data Engineering, April 2015, pp.66-77.

[24] Liu J F, Yang J C, Xiong L, Pei J. Secure skyline queries on cloud platform. In Proc. the 33rd IEEE Int. Conf. Data Engineering, April 2017, pp.633-644.

[25] Goldreich O. Foundations of Cryptography: Volume 2, Basic Applications. Cambridge University Press, 2004.

[26] Samanthula B K, Rao F Y, Bertino E, Yi X. Privacypreserving protocols for shortest path discovery over outsourced encrypted graph data. In Proc. Int. Conf. Information Reuse and Integration, August 2015, pp.427-434.

[27] Nikolaenko V, Ioannidis S, Weinsberg U, Joye M, Taft N, Boneh D. Privacy-preserving matrix factorization. In Proc. the ACM SIGSAC Conf. Computer \& Communications Security, November 2013, pp.801-812.
[28] Zhu H H, Meng X R, Kollios G. Privacy preserving similarity evaluation of time series data. In Proc. the 17th Int. Conf. Extending Database Technology, March 2014, pp.499510.

[29] Kolesnikov V, Sadeghi A R, Schneider T. Improved garbled circuit building blocks and applications to auctions and computing minima. In Proc. the 8th Int. Conf. Cryptology and Network Security, December 2009, pp.1-20.

[30] Mokbel M F, Chow C Y, Aref W G. The new Casper: Query processing for location services without compromising privacy. In Proc. the 32nd Int. Conf. Very Large Data Bases, September 2006, pp.763-774.

[31] Huang Y, Evans D, Katz J, Malka L. Faster secure twoparty computation using garbled circuits. In Proc. the 20th USENIX Conf. Security, August 2011.

[32] Li G L, Wang J N, Zheng Y D, Franklin M J. Crowdsourced data management: A survey. IEEE Trans. Knowledge and Data Engineering, 2016, 28(9): 2296-2319.

[33] Chen L, Shahabi C. Spatial crowdsourcing: Challenges and opportunities. Bulletin of the Technical Committee on Data Engineering, 2016, 39(4): 14-25.

[34] Tong Y X, She J Y, Ding B L, Wang L B, Chen L. Online mobile micro-task allocation in spatial crowdsourcing. In Proc. the 32nd IEEE Int. Conf. Data Engineering, May 2016, pp.49-60.

[35] Zheng L, Chen L. Mutual benefit aware task assignment in a bipartite labor market. In Proc. the 32nd IEEE Int. Conf. Data Engineering, May 2016, pp.73-84.

[36] Yiu M L, Ghinita G, Jensen C S, Kalnis P. Enabling search services on outsourced private spatial data. The $V L D B$ Journal, 2010, 19(3): 363-384.

[37] Yao B, Li F F, Xiao X K. Secure nearest neighbor revisited. In Proc. the 29th IEEE Int. Conf. Data Engineering, April 2013, pp.733-744.

[38] Shin M, Cornelius C, Peebles D, Kapadia A, Kotz D, Triandopoulos N. AnonySense: A system for anonymous opportunistic sensing. Pervasive and Mobile Computing, 2011, $7(1): 16-30$.

[39] Boutsis I, Kalogeraki V. Privacy preservation for participatory sensing data. In Proc. IEEE Int. Conf. Pervasive Computing and Communications, March 2013, pp.103-113.

[40] Agir B, Papaioannou T G, Narendula R, Aberer K, Hubaux $\mathrm{J}$ P. User-side adaptive protection of location privacy in participatory sensing. GeoInformatica, 2014, 18(1): 165-191.

[41] Vergara-Laurens I J, Mendez D, Labrador M A. Privacy, quality of information, and energy consumption in participatory sensing systems. In Proc. IEEE Int. Conf. Pervasive Computing and Communications, March 2014, pp.199-207.

[42] Zhang F, He L, He W B, Liu X. Data perturbation with state-dependent noise for participatory sensing. In Proc. IEEE INFOCOM, March 2012, pp.2246-2254.

[43] Li Q H, Cao G H. Efficient and privacy-preserving data aggregation in mobile sensing. In Proc. the 20th IEEE Int. Conf. Network Protocols, October 30-November 2, 2012.

[44] Kazemi L, Shahabi C. A privacy-aware framework for participatory sensing. ACM SIGKDD Explorations Newsletter, 2011, 13(1): 43-51. 


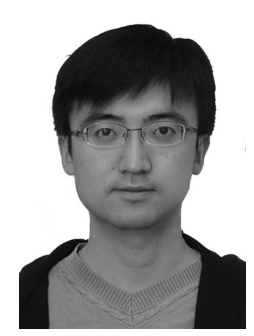

An Liu is an associate professor in the School of Computer Science and Technology at Soochow University, Suzhou. He received his Ph.D. degree in computer science from both City University of Hong Kong (CityU), Hong Kong, and University of Science and Technology of China (USTC), Hefei, in 2009. His research interests include spatial databases, crowdsourcing, data security and privacy, and cloud/service computing. He has published more than 80 papers in referred journals and conferences, including IEEE TKDE, IEEE TSC, GeoInformatica, KAIS, ICDE, WWW, etc. He served as the Workshop Co-Chairs of WISE 2017 and DASFAA 2015. He is on the reviewer board of several top journals such as IEEE TKDE, IEEE TSC, IEEE TII, IEEE TCC, ACM TOIT, JSS, DKE, FGCS, WWWJ, and JCST.

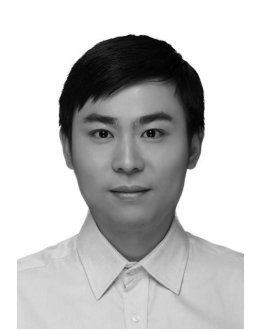

Zhi-Xu Li is an associate professor in the School of Computer Science and Technology at Soochow University, Suzhou. He worked as a research fellow at King Abdullah University of Science and Technology, Thuwal. He received his $\mathrm{Ph} . \mathrm{D}$. degree in computer science from the University of Queensland, Queensland, in 2013, and his B.S. and M.S. degrees in computer science from Renmin University of China, Beijing, in 2006 and 2009 respectively. His research interests include data cleaning, big data applications, information extraction and retrieval, machine learning, deep learning, knowledge graph, and crowdsourcing.

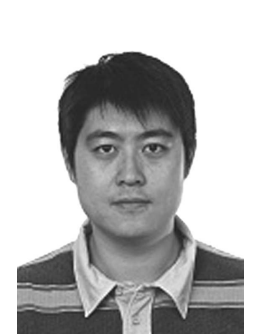

Guan-Feng Liu is an associate professor in the School of Computer Science and Technology at Soochow University, Suzhou. He received his $\mathrm{Ph} . \mathrm{D}$. degree in computer science from Macquarie University, Sydney, in 2013. His research interests include social network mining and trust. He has published over 40 papers in the most prestigious journals and conferences.

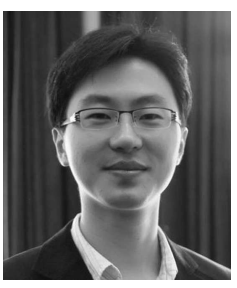

Kai Zheng is a professor in the School of Computer Science and Technology at Soochow University, Suzhou. He received his Ph.D. degree in computer science from University of Queensland, Queensland, in 2012. His research interests include finding effective and efficient solutions for managing, integrating and analyzing big data for business, scientific and personal applications. He has been working in the area of spatialtemporal databases, uncertain databases, trajectory computing, social-media analysis, and bioinformatics. He has published over 60 papers in the highly referred journals and conferences such as SIGMOD, ICDE, EDBT, The VLDB Journal, ACM Transactions, and IEEE Transactions.

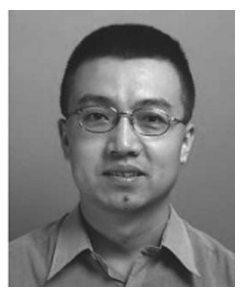

Min Zhang is a distinguished professor in the School of Computer Science, Soochow University, Suzhou. He received his Bachelor's degree and Ph.D. degree in computer science from Harbin Institute of Technology, Harbin, in 1991 and 1997, respectively. His current research interests include machine translation, natural language processing, and artificial intelligence.

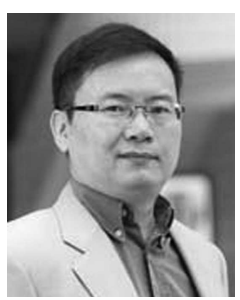

Qing Li received his B.E. degree from Hunan University, Changsha, and M.S. and Ph.D. degrees from the University of Southern California, Los Angeles, all in computer science. $\mathrm{He}$ is currently a professor with the Department of Computer Science, City University of Hong Kong, Hong Kong. His current research interests include dynamic object modeling, multimedia and mobile information retrieval and management, distributed databases and data warehousing/mining, and workflow management and web services.

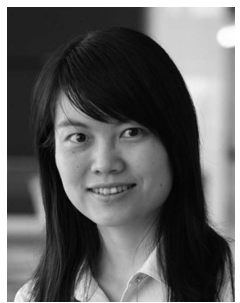

Xiangliang Zhang is an associate professor and directs the Machine Intelligence and kNowledge Engineering (MINE) Laboratory in King Abdullah University of Science and Technology (KAUST), Thuwal. She earned her Ph.D. degree in computer science from INRIA-University Paris-Sud 11, France, in 2010. Her main research interests and experiences are in diverse areas of machine learning and data mining. She has published over 70 papers in referred journals and conferences, including TKDE, SIGKDD, VLDB J, AAAI, IJCAI, ICDM, ECML/PKDD, CIKM, InfoCom, etc. 